\title{
The Canis Major Dwarf Galaxy
}

\author{
Geraint F. Lewis ${ }^{\mathrm{A}, \mathrm{E}}$, Rodrigo A. Ibata ${ }^{\mathrm{B}}$, Michael J. Irwin ${ }^{\mathrm{C}}$, Nicolas F. Martin ${ }^{\mathrm{B}}$, \\ Michele Bellazzini ${ }^{\mathrm{D}}$, and Blair Conn ${ }^{\mathrm{A}}$ \\ A Institute of Astronomy, School of Physics, A29, University of Sydney, Sydney NSW 2006, Australia \\ B Observatoire de Strasbourg, 11 Rue de l'Universite, 6700 Strasbourg, France \\ ${ }^{\mathrm{C}}$ Institute of Astronomy, Madingley Road, Cambridge, CB3 OHA, UK \\ D INAF-Osservatorio Astronomico di Bologna, Via Ranzani 1, 40127 Bologna, Italy

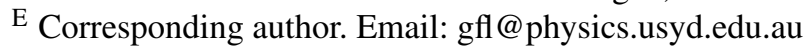

Received 2004 May 2, accepted 2004 August 10

\begin{abstract}
Recent observational evidence suggests that the Sagittarius dwarf galaxy represents the only major ongoing accretion event in the Galactic halo, accounting for the majority of stellar debris identified there. This paper summarises the recent discovery of another potential Milky Way accretion event, the Canis Major dwarf galaxy. This dwarf satellite galaxy is found to lie just below the Galactic plane and appears to be on an equatorial orbit. Unlike Sagittarius, which is contributing to the Galactic halo, the location and eventual demise of Canis Major suggests that it represents a building block of the thick disk.
\end{abstract}

Keywords: Galaxy: structure — Galaxy: evolution — galaxies: dwarf

\section{Introduction}

$\Lambda \mathrm{CDM}$ represents the current paradigm for the formation and evolution of structure in the Universe. While successul on large scales, focus has recently turned to its inability to correctly predict the number of satellite systems in galactic halos (Klypin et al. 1999). This missing satellite problem is apparent with the Milky Way, with recent observations indicating that the Milky Way has undergone a single large accretion, the Sagittarius Dwarf galaxy, in the last $\sim 7 \mathrm{Gyr}$ (Ibata et al. 2002; Majewski et al. 2003), although some older accretions are apparent in phase-space (Helmi et al. 1999; Brook et al. 2003).

To compare with predictions from $\Lambda \mathrm{CDM}$ models, the accretion history of the Milky Way needs to be mapped out. As with many studies of the Universe, however, our view of the Galactic halo, and any accreting systems, is obscured by the disk of the Milky Way. Recent large scale surveys of this region, however, have uncovered a curious overdensity of stars that has been interpreted as being part of a current accretion event that is taking place within the plane of the Milky Way. This paper reviews the discovery of this object and our current understanding of its properties.

\section{The Ring around the Galaxy}

The first indication of an additional halo population of stars was found by Newberg et al. (2002) while examining halo stars drawn from the Sloan Digital Sky Survey. Taken in a narrow band of $\sim 2.5^{\circ}$ about the celestial equator, and selected to have the colours of F-stars, these data revealed a prominent overdensity of halo stars which has been interpreted as the survey slicing through the stream of tidal debris from the Sagittarius Dwarf Galaxy. One additional prominent overdensity of stars was identified within the halo, towards the Galactic anti-centre, in the direction of Monoceros, at a galactocentric distance of $\sim 18 \mathrm{kpc}$ and width $<6 \mathrm{kpc}$. While a Galactic origin could not be conclusively ruled out, Newberg et al. (2002) suggested that this too represented a tidal stream, but of a yet unknown disrupting companion galaxy.

Spurred by this discovery, Ibata et al. (2003) searched for the signature of the Monoceros stream of stars in the Isaac Newton Telescope Wide Field Survey data archive. Analysis of colour-magnitude diagrams (Ibata et al. 2003) confirmed the identification of the distinct stellar population in the vicinity of Monoceros. This study also identified the Monoceros stream population in a number of additional fields, revealing this population to extend $\sim 100^{\circ}$ over the sky, within $\sim 30^{\circ}$ of the Galactic equator, and Ibata et al. (2003) suggest that the Monoceros stream actually rings the Galaxy. Fitting the main sequence of this stellar population in each field, this study estimates that the distance to the stream ranges from $\sim 15$ to $\sim 20 \mathrm{kpc}$, with an apparent scale-height of $\sim 0.75 \mathrm{kpc}$. While they consider the possibility that this stellar population was an accreting dwarf, they also pointed out that their data is consistent with other hypotheses including an outer spiral arm or unknown flare/warp generated via a resonance in the Galactic disk.

At the same time as the Ibata et al. (2003) results, Yanny et al. (2003) presented a kinematic analysis of a Galactic halo stars drawn from the SDSS. Focussing upon F-star candidates, this study obtained spectra in several regions, allowing the determination of their kinematic properties. Accounting for the Galactic contribution, the 
Monoceros population was found to have a velocity dispersion of $\sim 25-30 \mathrm{~km} \mathrm{~s}^{-1}$. While of similar order to the tidal debris torn from the Sagittarius dwarf, this velocity dispersion is quite distinct from the spheroid, thick disk or any known warp or flare. From these velocities, Yanny et al. (2003) deduce that the orbital velocity of the stream of stars is prograde and (assuming circular orbits) is $215 \pm 25 \mathrm{~km} \mathrm{~s}^{-1} 1$, and that the stars appear to be relatively metal-poor $([\mathrm{Fe} / \mathrm{H}]=-1.6)$. Yanny et al. (2003) conclude by proposing a simple model for the Monoceros stream as a disrupting dwarf, orbiting the Milky Way at a distance of $\sim 18 \mathrm{kpc}$; the dwarf galaxy's stars are undulating above and below the plane of the Milky Way by $\sim 6 \mathrm{kpc}$.

Several additional programs have focused upon the Monoceros stream ${ }^{2}$. Using M-giant star candidates drawn from 2MASS, Rocha-Pinto et al. (2003) further confirmed the existence of the Monoceros Stream as a distinct stellar population beyond the edge of the disk of the Milky Way. With a galactocentric distance of $18 \pm 2 \mathrm{kpc}$, they find the arc of material possesses an angular extent of at $\sim 170^{\circ}$, with the presence of M-giants indicating the stellar population of the Monoceros stars possesses a higher metallicity than previously estimated $([\mathrm{Fe} / \mathrm{H}] \sim 0.4 \pm 0.3)$. Crane et al. (2003) extended this work, obtaining velocities of 2MASS selected stars over $\sim 100^{\circ}$ of the Monoceros stream. While confirming a velocity dispersion of $\sim 20 \mathrm{~km} \mathrm{~s}^{-1}$, these data also indicate the stars in the Monoceros stream orbit the Galaxy in a prograde fashion, with little eccentricity. While this is somewhat worrisome for the tidally disrupting dwarf galaxy hypothesis, Crane et al. (2003) point out that such features are apparent in numerical simulations of in-plane dwarf accretion (Helmi et al. 2003). Furthermore, this study identifies four globular clusters that are spatially and kinematically aligned with the putative stellar stream; such an alignment argues against a Galactic origin for the stream, such as a spiral arm. Finally, Frinchaboy et al. (2004) noted that five globular clusters aligned with the Monoceros stream, as well as $\sim 15$ outer, old stellar clusters that may also be part of the same population; these clusters lie in a plane which is significantly tilted $\left(\sim 17^{\circ}\right)$ to that of the Milky Way. The population of globular clusters is reminiscent of the Sagittarius Dwarf galaxy which has appears to have deposited a similar number of globular clusters into the halo of the Milky Way, bolstering the argument that the Monoceros stream represents a similarly disrupting dwarf galaxy.

The extensive nature of the material strongly suggested that its origin lay in a disrupting system, in an event similar to that of Sagittarius, but occurring in the plane of the Galaxy. This, however, was not the only explanation for its origin as it may represent a previously unidentified aspect

\footnotetext{
${ }^{1}$ An erratum to the original result of $110 \pm 25 \mathrm{~km} \mathrm{~s}^{-1}$ was presented in Yanny et al. (2004).

${ }^{2}$ The stream of stars has acquired several names since its discovery. For the sake of consistency, in this paper it is referred to solely as the Monoceros Stream.
}

of Galactic structure. If the disrupting system hypothesis is correct, and its destruction is not yet complete, we should expect to identify some remnant of the original dwarf galaxy. However, the expected location, observed against the plane of the Galaxy, makes the detection of such a remnant difficult.

\section{The Canis Major Dwarf}

As with Rocha-Pinto et al. (2003), Martin et al. (2004) employed 2MASS to search for a signature of Monoceros stream of stars. Pushing the search to $|b| \sim 5^{\circ}$, Martin et al. (2004) mapped the density of M-giant stars around the Galactic equator. This reveals a strong asymmetry about the Galactic plane which is interpreted as being the Monoceros ring of stars snaking around the outskirts of the Milky Way. Several prominent features are noted, including a strong Northern arc and weaker Southern arc, both of which extend for more than $\sim 100^{\circ}$ on the sky (see Figure 1).

Intriguingly, Martin et al. (2004) also identified a strong, elliptical overdensity of M-stars at $(l, b)=$ $\left(240^{\circ},-8^{\circ}\right)$, aligned somewhat with the Galactic disk. The (heliocentric) distance to this stellar overdensity is $D_{\odot}=7.1 \pm 0.1 \mathrm{kpc}$, with a major axis is $\sim 4.2 \mathrm{kpc}$. With $\sim 2300 \mathrm{M}$-giant stars within $10^{\circ}$ of its centre, this overdensity contains a similar number of M-giants to the Sagittarius Dwarf galaxy, a system which we know is currently being cannibalised by the Milky Way. Given that this implies that the mass of the Canis Major overdensity is $\sim 10^{8}-10^{9} M_{\odot}$, Martin et al. (2004) concluded that it too represents a dwarf galaxy also undergoing tidal disruption, and possibly representing the progenitor of the Monoceros stream of stars.

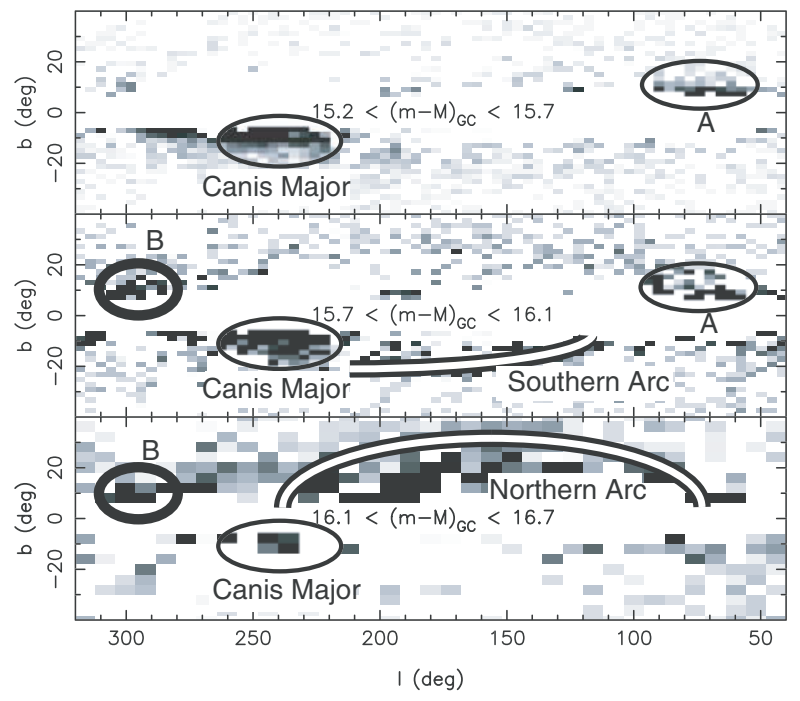

Figure 1 Binned M-giant counts about the Galactic plane. The three panels denote different magnitude (and hence distance) ranges, with the galactocentric distance modulus labelled upon each panel. Also in each panel, the counts represent the asymmetry between the north and south (i.e. southern data subtracted from the north, and vice versa). The main body of Canis Major and prominent structures in the M-giant distribution are noted (fig. 5 from Martin et al. 2004). 
Unlike Sagittarius, which passes over the poles of the Milky Way, the identification of the Canis Major dwarf galaxy (as this overdensity will now be referred to) represents the first detection of an accretion occurring within the plane of the Galaxy.

Martin et al. (2004) also noted that possibly five globular clusters were associated in phase-space with the Canis Major dwarf galaxy. This is a similar number to the globular cluster population currently associated with the Sagittarius dwarf galaxy, providing further evidence for the origin of Canis Major. Furthermore, Martin et al. (2004) noted the phase-space grouping of a number of four open galactic clusters directly associated with the main body of Canis Major ${ }^{3}$.

In an attempt to understand the observed distribution of M-stars, Martin et al. (2004) also undertook a series of numerical simulations. Utilising detailed models for the mass distribution of the Milky Way (Dehnen \& Binney 1998), these simulations involved following the dynamical dissociation of dwarf galaxies (modelled as King profiles) as they orbited the Galaxy. The results of this procedure favoured a dwarf galaxy with an initial mass of $\sim 5 \times 10^{8} M_{\odot}$, with a orbital period of $\sim 0.4 \mathrm{Gyr}$. While such simulations can reasonably account for the observed distribution of M-stars not only in the body of Canis Major, but also along the extensive arcs above and below the Galactic plane, the current data does not allow a definitive differentiation between prograde and retrograde orbits. Martin et al. (2004), however, point out that the resultant orbits of the debris in such an encounter closely mimic those of stars in the thick disk. Given that the estimated mass of this single dwarf is roughly $\sim 10 \%$ of the entire thick disk, then the thick disk could be formed via only a few such accretion events.

Further observational evidence for the nature of the Canis Major dwarf came from the study of Bellazzini et al. (2004) who identified the main-sequence and red giant branch populations of Canis Major in the background to the Galactic open clusters NGC2477, Tombaugh 1, and Berkeley 33. Analysis of this population suggested it is somewhat metal-rich $(-0.7<[\mathrm{Fe} / \mathrm{H}]<0.0)$, with an age of 2-7 Gyr, although an apparent blue plume of stars is taken as evidence of a more recent episode of star formation. This study also finds a photometric parallax for the main body of Canis Major of $\sim 8.3 \pm 1.2 \mathrm{kpc}$, larger than the M-giant study of Martin et al. (2004), whose smaller distance determination they put down to poorer systematics in the M-giant photometric parallax. Finally, Bellazzini et al. (2004) also suggest that as well as the identified globular cluster population, two old open clusters, AM-2 and Tombaugh 2, are possibly associated with the Canis Major dwarf.

Utilising the Second U.S. Naval Observatory CCD Astrograph Catalog (UCAC2), Ibata et al. (2004) examined the proper motions of stars in the vicinity of the

\footnotetext{
${ }^{3}$ Several of these clusters were also noted by Frinchaboy et al. (2004) as being part of the Monoceros stream.
}

Canis Major dwarf galaxy. Cross-identifying M-giant stars drawn from the 2MASS catalog, the Galacticlongitudinal motion of Canis Major was found to be

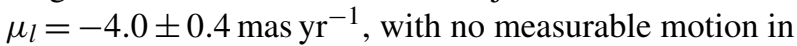
Galactic latitude. At a distance of $D=8.3 \mathrm{kpc}$, this corresponds to transverse velocity of $\sim 238 \pm 28 \mathrm{~km} \mathrm{~s}^{-1}$, with Canis Major on a prograde orbit about the Milky Way. Since this value is higher than that predicted by Martin et al. (2004), drawn from their numerical simulation, it is clear the full three-dimensional velocity of Canis Major will be required for the detailed numerical modelling of this accretion event.

Recently, Forbes et al. (2004) further examined the globular cluster population associated with the Canis Major dwarf galaxy, finding that the age-metallicity relationship for these is distinct from the main globular cluster population of the Milky Way. Furthermore, these globular clusters are somewhat smaller than expected if they were drawn from the Galactic population. Both these lines of evidence add further weight to the possibility that the globular clusters, and Canis Major itself, were formed

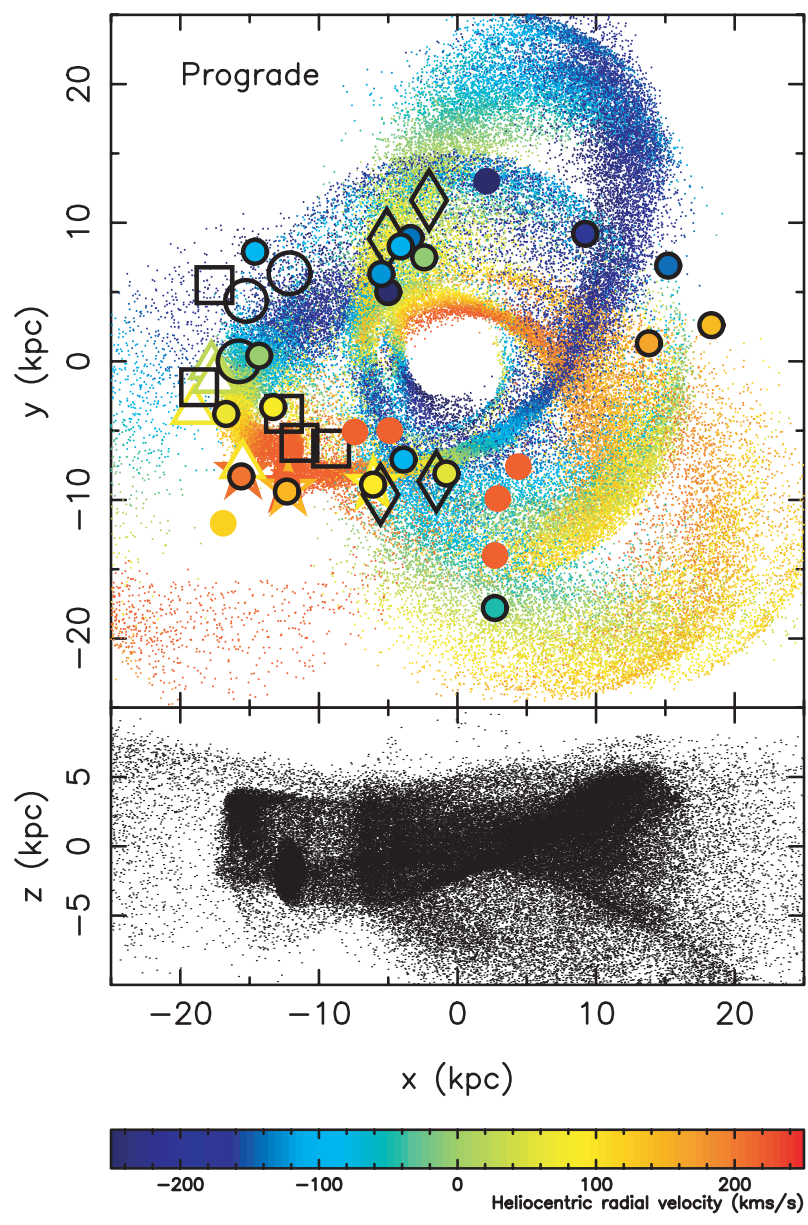

Figure 2 A numerical simulation of the demise of the Canis Major dwarf galaxy. The top panel presents the view from above the Milky Way [with the Sun located at $(x, y, z)=-8,0,0 \mathrm{kpc}$ ]. The lower panel presents the side-on view of the debris. The colour coding in both denoted the heliocentric velocity of the stars (as given in the lower key), while the symbols represent data from the INT survey and 2MASS (fig. 14 from Martin et al. 2004). 
elsewhere, were drawn in presumably as the initial orbit decayed by dynamical friction, and represent an on-going accretion event onto the Milky Way galaxy.

\section{Further Observations}

While the tidally disrupting dwarf galaxy is the favoured explanation for the observed overdensity of star in Canis Major, current observations are not yet completely conclusive, and the possibility that the Monoceros Ring and Canis major dwarf galaxy actually represent some unknown aspect of Galactic structure has not been ruled out. Hence, several observational programs are underway to address this issue.

The first is an extension of the earlier study by Ibata et al. (2003), systematically mapping above and below the Galactic plane with wide-field camera observations. This has been completed in the north, using the Wide-Field Camera on the Isaac Newton Telescope; these data are being analysed and will be published shortly (Conn et al., in preparation). The southern survey, with the Wide-Field Imager at the Anglo-Australian Telescope, is currently underway and will be completed by mid-2004. These will provide important probes of the extent of the stellar material, as well as constraining its distance (via main sequence fitting).

To augment these studies, $2 \mathrm{dF}$ observations of the main body of Canis Major and the extensive stream of stars are being conducted to obtain stellar kinematics, by means of the calcium triplet, over $\sim 100^{\circ}$ of the sky. Coupled with the spatial data obtained with the wide-field camera surveys, these data should test the hypotheses that Canis Major represents a truly disrupting dwarf galaxy, or (equally interesting) is a currently unknown aspect of Galactic structure. Furthermore, if the dwarf galaxy is confirmed, then these data will provide important constraints to numerical simulations of its orbit and eventual demise.

\section{Acknowlegements}

G.F.L. thanks Joss Bland-Hawthorn for inviting him to the Little Bay Meeting. The anonymous referee is thanked for their constructive comments.

\section{References}

Bellazzini, M., Ibata, R., Monaco, L., Martin, N., Irwin, M. J., \& Lewis, G. F. 2004, MNRAS, in press, astro-ph/0311119

Brook, C. B., Kawata, D., Gibson, B. K., \& Flynn, C. 2003, ApJ, 585, L125

Crane, J. D., Majewski, S. R., Rocha-Pinto, H. J., Frinchaboy, P. M., Skrutskie, M. F., \& Law, D. R. 2003, ApJ, 594, L119

Dehnen, W., \& Binney, J. 1998, MNRAS, 294, 429

Forbes, D. A., Strader, J., \& Brodie, J. P. 2004, AJ, 127, 3394

Frinchaboy, P. M., Majewski, S. R., Crane, J. D., Reid, I. N., Rocha-Pinto, H. J., Phelps, R. L., Patterson, R. J., \& Muñoz, R. R. 2004, ApJ, 602, L21

Helmi, A., Navarro, J. F., Meza, A., Steinmetz, M., \& Eke, V. R. 2003, ApJ, 592, L25

Helmi, A., White, S. D. M., de Zeeuw, P. T., \& Zhao, H. 1999, Natur, 402,53

Ibata, R. A., Irwin, M. J., Lewis, G. F., Ferguson, A. M. N., \& Tanvir, N. 2003, MNRAS, 340, L21

Ibata, R., Bellazzini, M., Irwin M., Lewis G. F., \& Martin, N. F. 2004, MNRAS, submitted

Ibata, R. A., Lewis, G. F., Irwin, M. J., \& Cambrésy, L. 2002, MNRAS, 332, 921

Klypin, A., Kravtsov, A. V., Valenzuela, O., \& Prada, F. 1999, ApJ, 522,82

Majewski, S. R., Skrutskie, M. F., Weinberg, M. D., \& Ostheimer, J. C. 2003, ApJ, 599, 1082

Martin, N. F., Ibata, R. A., Bellazzini, M., Irwin, M. J., Lewis, G. F., \& Dehnen, W. 2004, MNRAS, 348, 12

Newberg, H. J., et al. 2002, ApJ, 569, 245

Rocha-Pinto, H. J., Majewski, S. R., Skrutskie, M. F., \& Crane, J. D. 2003, ApJ, 594, L115

Yanny, B., et al. 2003, ApJ, 588, 824

Yanny, B., et al. 2004, ApJ, 605, 575 